\title{
Study of Gas Heating by a Microwave Plasma Torch*
}

\author{
Katell Gadonna $^{1}$, Oliviez Leroy ${ }^{1}$, Philippe Leprince ${ }^{1}$, Luis Lemos Alves ${ }^{2}$, Canoline Boisse-Laporte ${ }^{1}$ \\ ${ }^{1}$ Laboratoire de Physique des Gaz et des Plasmas, UMR 8578 CNRS, Université Paris-Sud, Orsay, France \\ ${ }^{2}$ Instituto de Plasmas e Fusão Nuclear, Instituto Superior Técnico, Universidade Técnica de Lisboa, Lisboa, Portugal \\ Email: olivier.leroy@u-psud.fr
}

Received August 26, 2012; revised September 24, 2012; accepted October 2, 2012

\begin{abstract}
Among the different types of microwave plasma torches, the axial injection torch (TIA) has been used for several years to create chemically active species, in applications such as gas analysis, surface processing and gaseous waste treatments. The TIA allows the coupling of microwave energy $(2.45 \mathrm{GHz})$ to a gas injected axially at the nozzle's exit. The TIA produces non-local thermodynamic equilibrium plasmas with a high luminosity and a maximum density of charged particles at the nozzle's exit. The present work is dedicated to study the plasma created by a TIA running at atmospheric pressure. The study involves both experiment and modeling of this torch, in order to maximize the coupling between the microwave power and the plasma and to define the optimum plasma and flow operating conditions for plasma-to-gas heat transfer.
\end{abstract}

Keywords: Microwave Plasma Torch; Non-Equilibrium Plasma; Atmospheric Pressure; Thermal Transfer; Flow Modeling

\section{Introduction}

The operation of microwave plasma torches (MPT) ensures a strong gas ionization due to an efficient microwave coupling, with potential interest in different applications [1], generating also a considerable amount of thermal energy that can be used to heat large volumes of gas, for example to adjust the altitude of dirigible balloons. These balloons have a renewed interest considering their possibility to operate vertically during takeoff and landing procedures, thus without the need for dedicated infrastructures, and their ability to transport heavy materials [2].

Unlike resistors, which are commonly used to heat gases by contact with hot surfaces, heating by MPTs relies on the distribution of the electromagnetic fields existing within the plasma and leading to its partial ionization. Therefore, MPT-assisted heating is volumetric and efficient (yielding gas temperatures $T_{g}$ that can reach 2000 to $4000 \mathrm{~K}$ [1]), allowing local rapid $T_{g}$ variations especially for working gases like helium, with high thermal conductivity [3].

Microwave induced plasmas are created and maintained by using an electromagnetic energy source, with frequency in the range of $300 \mathrm{MHz}$ to $10 \mathrm{GHz}$, in the absence of electrodes, thus limiting gas contamination. These sources have a wide range of operating powers

\footnotetext{
${ }^{*}$ This work was partially supported by the Portuguese FCT-MCTES (Project PTDC/FIS/65924/2006).
}

(from a few watts up to $\sim 1 \mathrm{~kW}$ ), remaining below the high-power required by thermal torches [4], at pressures varying from $10^{-2} \mathrm{~Pa}$ to several $10^{5} \mathrm{~Pa}$. Microwave torches create non-LTE plasmas characterized by a strong ionization degree (electron densities $n_{e} \sim 10^{12}-10^{16} \mathrm{~cm}^{-3}$ ) and by different temperatures for the various constitutive species of the gas/plasma system (higher electron temperatures, $T_{e} \sim 12000-25000 \mathrm{~K}$, and lower neutral gas temperatures, $T_{g} \sim 1500-10000 \mathrm{~K}$ ) [1], thus favoring an energy flow from the plasma electrons to the gas excited species.

MPTs can operate in both confined $[5,6]$ and non-confined $[7,8]$ modes, corresponding to semi-metallic torches and metallic torches, respectively. In the confined mode the plasma is created within a quartz tube (transparent to microwaves), where the gas flows through an electromagnetic wave that settles generally in a cylindrical structure. This wave is a surface-wave, a plasma wavemode which creates the plasma medium while it propagates along it. In the non-confined mode the plasma is created at the outlet of the nozzle that terminates a coaxial waveguide (Figure 1). The gas flows inside the inner metallic tube of the coaxial structure, where a TEM mode propagates, and the electromagnetic field couples to the plasma at the nozzle's exit, where the gas exhausts. Non-confined plasma systems are more adapted to volumetric gas heating. Here we have chosen the well-known TIA ("Torche à Injection Axiale" or Axial Injection Torch), which couples the TEM wave through a rectan- 


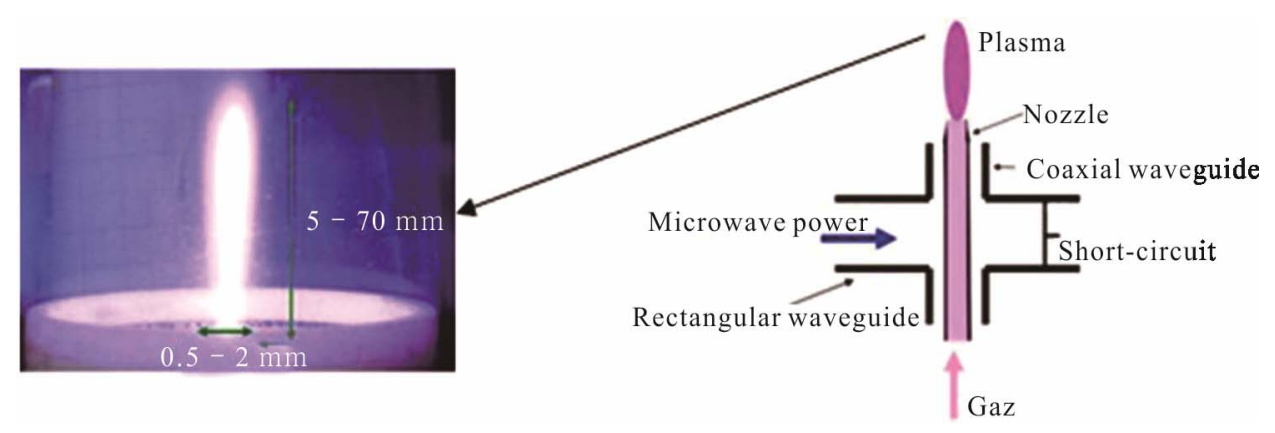

Figure 1. TIA design.

gular waveguide-coaxial line transition (Figure 1) [1] Note that the non-confined plasma created with this configuration can be launched either in open air or in a controlled atmosphere, for example by adapting some quartz tube or chamber to the system.

Since the 80 s, the TIA has been studied by different research groups (from the University of Montreal, the Technical University of Eindhoven and the University of Cordoba) that focus mainly on the experimental characterization of the plasma it creates $[7,9,10]$, paying also some attention to its electromagnetic and hydrodynamic modeling $[11,12]$. These studies have shown that the TIA could be efficient in applications such as analytical chemistry [13] (in relation also with its ability to create chemically active species), surface treatment [14] and gas treatment [15].

This work focuses on the plasma-to-gas thermal transfer, which is studied using both modeling and experiments. Modeling involves a twofold description of the TIA (electromagnetic and hydrodynamic), aiming to maximize the microwave power coupled to the system and to define the plasma and flow conditions that yield an optimum transfer of thermal power from the plasma to the gas. Experiments analyze the MPT efficiency and stability, as a function of both the input power and the input gas flow, providing a framework of conditions for the simulations. Values for the plasma parameters (electron density and temperature, used as input data to the model) and for the gas temperature (also at the wall, used as boundary condition in the model) were obtained mainly from optical emission spectroscopy (OES) diagnostics.

\section{Experimental Set-Up}

Figure 2 presents a schematic representation of the experimental set-up adopted here. Helium gas at atmospheric pressure is injected into the inner conductor of the coaxial waveguide and exits through its end nozzle. We have used two nozzles, with $0.5 \mathrm{~mm}$ and $2 \mathrm{~mm}$ diameters, to extend the range of gas flow from 0.5 to $10 \mathrm{~L} \cdot \mathrm{min}^{-1}$ (cf. Section 4.1).
The microwaves propagate through a circuit of rectangular waveguides after which they are transmitted to the coaxial line. The system operates at $f=2.45 \mathrm{GHz}$ excitation frequency, for an incident power ranging from $100 \mathrm{~W}$ to $1 \mathrm{~kW}$. The short circuit and the stubs (Figure 2) enable to optimize the transition between the rectangular waveguide and the coaxial structure, maximizing the power coupled to the system. The power delivered by the generator $P_{G}$ is controlled by a bi-directional coupler which measures the incident $P_{i n c}$ and the reflected $P_{r e f}$ powers, by redirecting a small part of these powers to a detector connected to a power meter. The power transmitted to the system $P_{\text {trans }}=P_{\text {inc }}-P_{\text {ref }}$ is then radiated $\left(P_{r a d}\right)$ or absorbed $\left(P_{a b s}\right)$ by the plasma electrons

$$
P_{a b s}=\frac{n_{e} e^{2} v}{m_{e}\left(v^{2}+\omega^{2}\right)} \frac{E_{0}{ }^{2}}{2}
$$

In Equation (1) $e$ and $m_{e}$ are the electron charge and mass, respectively, $v\left(\mathrm{~s}^{-1}\right) \sim 6.8 \times 10^{-8} N\left(\mathrm{~cm}^{-3}\right)[16]$ is the electron-neutral collision frequency (with $N$ the gas density), $\omega=2 \pi f, E_{0}$ is the amplitude of the wave electric field and $V$ is the plasma volume.

The plasma is launched into a quartz tube of $5 \mathrm{~cm}$ in diameter and $40 \mathrm{~cm}$ in length, with an open-end to prevent gas recirculation (thus favoring flow stability). The quartz tube limits the contamination of the flowing helium by the surrounding atmospheric air, providing more definite boundary conditions for the simulations (see Appendix).

The TIA produces high-luminosity plasmas with a maximum charged particle density at the nozzle's exit [8]. For the work conditions considered here, the plasma dimensions vary between $0.5 \mathrm{~mm}$ and $2 \mathrm{~mm}$ in diameter (nozzle sizes) and between $5 \mathrm{~mm}$ and $7 \mathrm{~cm}$ in length. The radiation emitted by the plasma is collected by two lenses into an optical fiber and then it is transmitted to a spectrometer (HR 460 Jobin-Yvon) for OES measurements (cf. Section 4.2 for a description of the optical diagnostics). The lenses enable to magnify the plasma by a factor between 2 and 4, allowing focusing a cross section of the plasma onto the fiber entrance slit, integrating about 3 


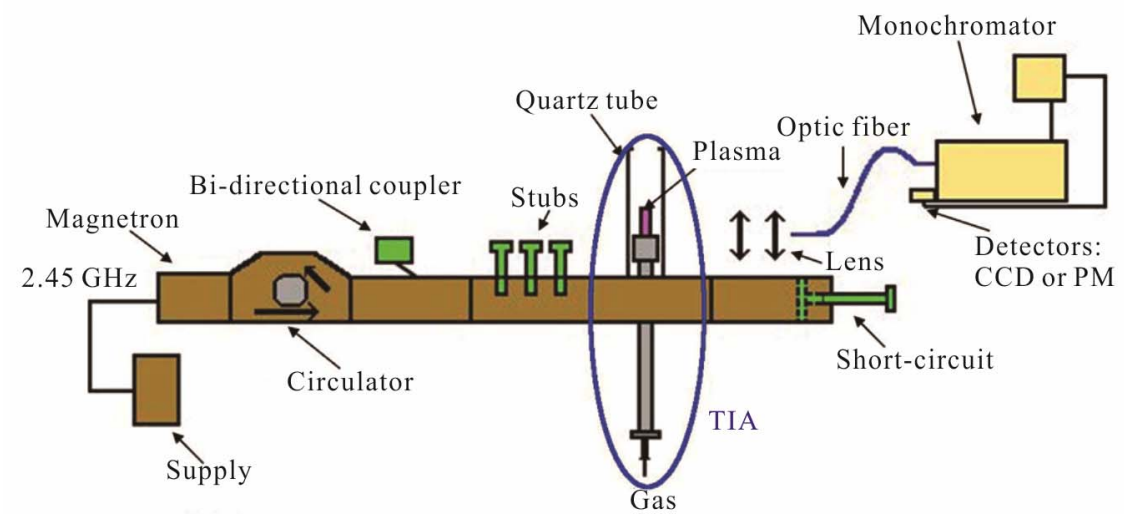

Figure 2. Schematic representation of the experimental set-up.

mm of plasma height. It can be seen that the plasma develops about $1 \mathrm{~mm}$ above the nozzle outlet.

\section{Modeling}

The complete modeling of a MPT requires the coupled description of its microwave excitation and the transport of charged and neutral particles in a flow regime. This goal can be achieved with the development of the following three independent but complementary simulation modules (Figure 3):

- An electromagnetic (EM) module which solves Maxwell's equations;

- A hydrodynamic (HD) module which solves NavierStokes' equations;

- A plasma (P) module which solves the electron and ion particle and energy transport equations.

Figure 3 schematizes the articulation between these calculation modules. The P-module receives as input data the information obtained from the EM-module, i.e. the electric field distribution $E$ and the power absorbed by the plasma $P_{a b s}$. Reciprocally, these quantities depend on the plasma parameters $\left(n_{e}, T_{e}, v\right)$, obtained as output from the P-module, via the plasma conductivity $\sigma$ [see also Equation (1)].

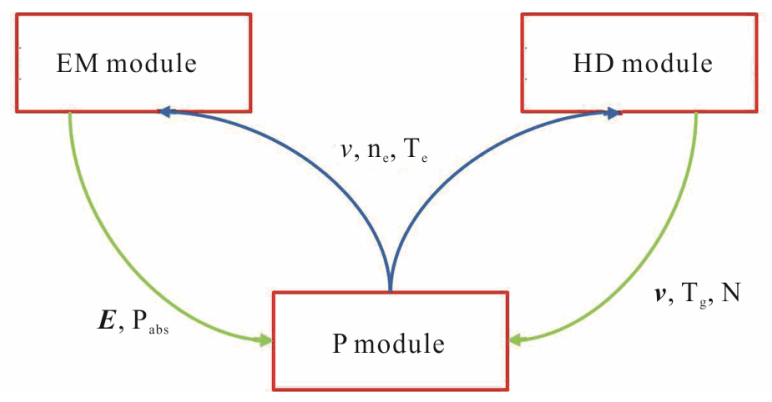

Figure 3. Modeling scheme of a MPT, based on the coupling of three independent simulation modules: an electromagnetic (EM) module, a hydrodynamic (HD) module and a plasma (P) module.

$$
\sigma=\frac{n_{e} e^{2}}{m_{e}(v+j \omega)}
$$

Here, the electron density and the electron-neutral collision frequency depend on the electron temperature $T_{e}$ and on the gas density $N$ (hence on its temperature $T_{g}$ ), which also affect the dynamics of the charged particles with the plasma. Therefore the HD-module output $\left(v, T_{g}\right.$, $N$ ), where $v$ is the gas flow velocity, are used as input to the P-module. Conversely, the plasma modifies the gas flow due to the ion drift and the gas heating, and so the plasma parameters are input to the HD-module.

Overall, the interlinked P, EM and HD features of the problem require an iterative solution between the corresponding calculation modules. This complexity is amplified by two extra difficulties: 1) the absence of closed boundaries defining the plasma geometry and 2) the multi-component features with the transport of species. The problem can nevertheless be simplified, by reducing its dimension to a 2D description (taking advantage of possible symmetries), and by imposing some work conditions to replace the self-consistent solution to the different modules.

In this work, we have developed the 3D EM and 2D HD modules, under the COMSOL Multiphysics platform [17], replacing the P-module (still in development) by some given conditions (both in profile and in intensity) for the plasma electron density and temperature. Details about the model equations, the calculation domains and the boundary conditions considered in this study are presented in appendix as supplementary information.

Our main interest concerns the heat transfer between the plasma and the gas, which justifies the investment in describing the electromagnetic and the hydrodynamic characteristics of MPTs. The goal is to define the work conditions (geometry, plasma, flow) which maximize 1) the power coupling between the microwave and the plasma and 2) the power transfer from the plasma to the gas, in order to obtain an efficient gas heating. 


\section{Experiments}

Information on the MPT efficiency and stability, as a function of both the input power and the input gas flow, were obtained experimentally. Measurements enabled to define a framework of input data for the simulations, providing also values for the plasma parameters and the gas temperature, to be used in the model.

\subsection{Work Conditions}

Figure 4 presents coupling efficiency diagrams, of $P_{\text {ref }} / P_{i n c}$ vs. the power delivered by the generator $P_{G}$ and the input gas flow $S$, for the $2 \mathrm{~mm}$ (Figure 4(a)) and $0.5 \mathrm{~mm}$ (Figure 4(b)) nozzle diameters used here. These diagrams define the operating conditions of the TIA. The grey areas correspond to the cases where no stable plasma is produced. In all the other areas, the plasma can be ignited with $P_{r e f} / P_{i n c}<10 \%$, i.e. power coupling (1$P_{\text {ref }}\left(P_{i n c}\right)>90 \%$. The red areas are the optimum operating conditions, with power coupling $>99.9 \%$. Measurements yield a very good power coupling ( $>90 \%$ ) for 400 to 800 $\mathrm{W}$ input power and 1 to $9 \mathrm{~L} \cdot \mathrm{min}^{-1}$ gas flow, and therefore the EM and the HD modeling will limit to these work conditions. Note that Reynolds' number is well below 2000 for these flows, which allows adopting a laminar regime in the HD description. The diagrams of Figure 4 reveal also the advantage in using a larger nozzle diameter, as it enables to operate the TIA over a more extended range of parameters.

Typically, a very good power coupling $(>90 \%)$ is obtained at gas flows between 5 and $9 \mathrm{~L} \cdot \mathrm{min}^{-1}$ for the 2 $\mathrm{mm}$ diameter nozzle and between 1 and $3 \mathrm{~L} \cdot \mathrm{min}^{-1}$ for the $0.5 \mathrm{~mm}$ diameter nozzle. These flows correspond to the average input gas velocities given in Table 1, where it is shown that the $2 \mathrm{~mm}$ diameter nozzle supports higher flow rates while the $0.5 \mathrm{~mm}$ nozzle induces higher velocities.

\subsection{Optical Diagnostics}

The gas temperature is estimated from the ro-vibrational spectrum of the 2nd positive system of nitrogen (incorporated from the air envelop surrounding the helium plas$\mathrm{ma}$ ), assuming that these molecules are in equilibrium with the plasma gas. The experimental spectra, recorded using a CCD detector with spectral resolution of $\sim 3 \AA$, are compared with simulations performed using the SPECAIR program [18]. Results yield $T_{g} \sim 1500-3000 \mathrm{~K}$.

The temperature on the wall of the $5 \mathrm{~cm}$ diameter quartz tube is measured by a thermocouple along its 40 $\mathrm{cm}$ length. A maximum value turns out to be around 600 $\mathrm{K}$ and located about $20 \mathrm{~cm}$ above the nozzle.

The electron density is deduced from Stark broadening measurements of the $H_{\beta}$ line $[19,20]$, recorded using a

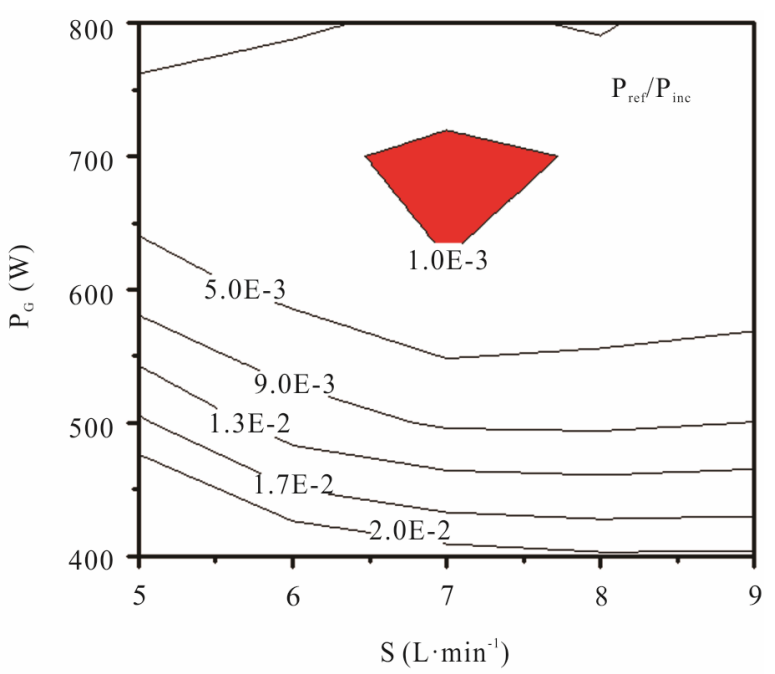

(a)

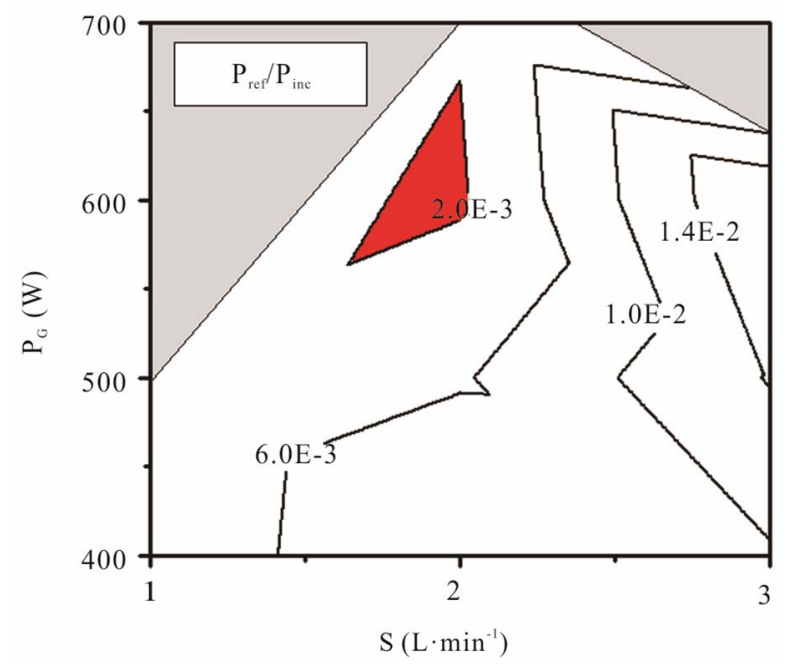

(b)

Figure 4. Coupling efficiency diagram, $P_{\text {ref }} / P_{\text {inc }}$ vs $\left(P_{G}, S\right)$, for TIAs with nozzle diameter of $2 \mathrm{~mm}$ (a) and $0.5 \mathrm{~mm}$ (b). $P_{G}$ is the microwave power in Watt delivered by the generator, $\boldsymbol{P}_{\text {ref }}$ and $\boldsymbol{P}_{\text {inc }}$ are respectively the reflected and incident power measured with a directional couplers and $S$ is the helium gas flow in $\mathrm{L} / \mathrm{min}$. The red areas signal the operating conditions for a microwave power coupling better than $99.9 \%$. The gray areas signal the operating conditions for which no stable plasma is produced.

Table 1. Gas flows and average velocities, for the nozzle diameters considered here.

\begin{tabular}{cccc}
\hline 2 mm diameter nozzle & \multicolumn{2}{c}{$0.5 \mathrm{~mm}$ diameter nozzle } \\
\hline $\begin{array}{c}\text { Flow } \\
\left(\mathrm{L} \cdot \mathrm{min}^{-1}\right)\end{array}$ & $\begin{array}{c}\text { Velocity } \\
\left(\mathrm{m} \cdot \mathrm{s}^{-1}\right)\end{array}$ & $\begin{array}{c}\text { Flow } \\
\left(\mathrm{L} \cdot \mathrm{min}^{-1}\right)\end{array}$ & $\begin{array}{c}\text { Velocity } \\
\left(\mathrm{m} \cdot \mathrm{s}^{-1}\right)\end{array}$ \\
\hline 5 & 27 & 1 & 85 \\
7 & 37 & 2 & 170 \\
9 & 48 & 3 & 255 \\
\hline
\end{tabular}


PM detector (spectral resolution of $\sim 1 \AA$ ). Results yield $n_{e} \sim 2 \times 10^{14}-4 \times 10^{14} \mathrm{~cm}^{-3}$ for the $2 \mathrm{~mm}$ nozzle and $n_{e} \sim$ $1 \times 10^{15}-1.5 \times 10^{15} \mathrm{~cm}^{-3}$ for the $0.5 \mathrm{~mm}$ nozzle, consistent with values obtained in previous studies [8-15]. The electron temperature could not be measured due to the experimental lack of precision. We estimate $T_{e} \sim 17,000$ $25,000 \mathrm{~K}$, according to the work of Torres et al. [21] and Jonkers et al. [10].

\section{Results}

\subsection{Electromagnetic Module}

The EM-module was used to study the interaction between the applied microwave field and the plasma. As mentioned in Section 3, the calculations were made imposing the plasma conditions (values and profiles for the electron density and temperature), which are given in the appendix. These conditions agree with our experimental observations and are also supported by other works [9, $11,21]$. The plasma impedance changes with $n_{e}$ and $v$ (see Equation (2)) [22], thus affecting the wave-plasma power coupling. Experimentally, after the plasma ignition the system can return to optimum power coupling conditions, at fixed frequency, by adjusting the short-circuit position. In the simulations, it is easier to work for a given excitation structure (hence a fixed short-circuit position) to obtain a frequency response at various maximum electron densities $n_{e \mathrm{MAX}}$.

The frequency response of the power transferred to the TIA is given in Figure 5 for $n_{e \mathrm{MAX}}=3 \times 10^{14}-12 \times$ $10^{14} \mathrm{~cm}^{-3}$. The plasma dimensions $(2 \mathrm{~mm}$ in diameter and $3 \mathrm{~cm}$ in length) correspond to those obtained with the $2 \mathrm{~mm}$ diameter nozzle. The first observation from this figure is that, for a device tuned in vacuum at $2.45 \mathrm{GHz}$

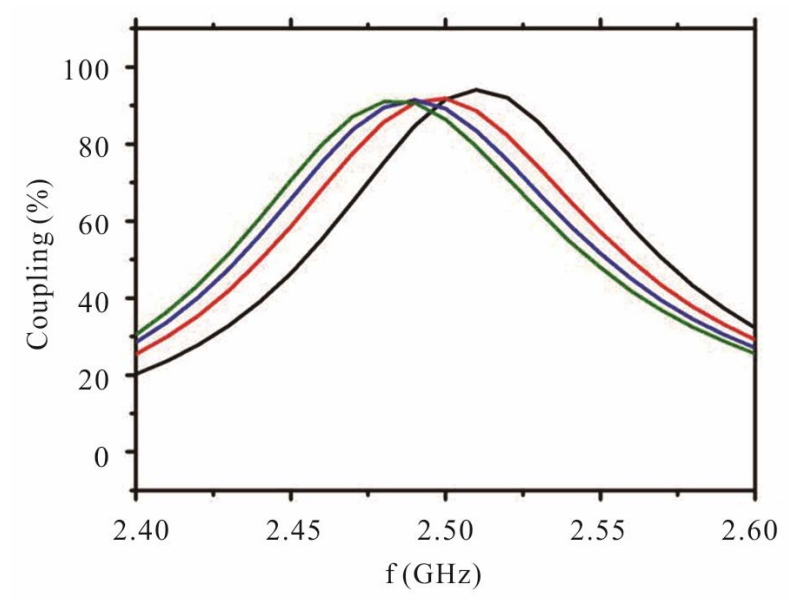

Figure 5. Power coupled to the TIA as a function of the excitation frequency, for the following maximum electron densities (in $10^{14} \mathrm{~cm}^{-3}$ ): 3 (black curve), 6 (red), 9 (blue) and 12 (green) and for plasma dimensions of $2 \mathrm{~mm}$ diameter and $3 \mathrm{~cm}$ length. excitation frequency, the power coupling remains very good (more than $90 \%$ in all the studied cases), regardless the maximum electron density imposed. Note that although the resonance frequency is above $2.45 \mathrm{GHz}$ with the plasma on, the maximum coupled power is shifted to lower frequencies as $n_{e \mathrm{MAX}}$ increases.

Figure 6(a) shows the spatial distribution of the timeaveraged Poynting vector intensity, corresponding to the flux of electromagnetic energy carried by the excitation wave. Note that in the central region of the device the Poynting vector is directed towards the plasma, which absorbs most of the energy. The remaining part of the energy is radiated.

Figure 6(b) plots the power absorbed by the plasma as

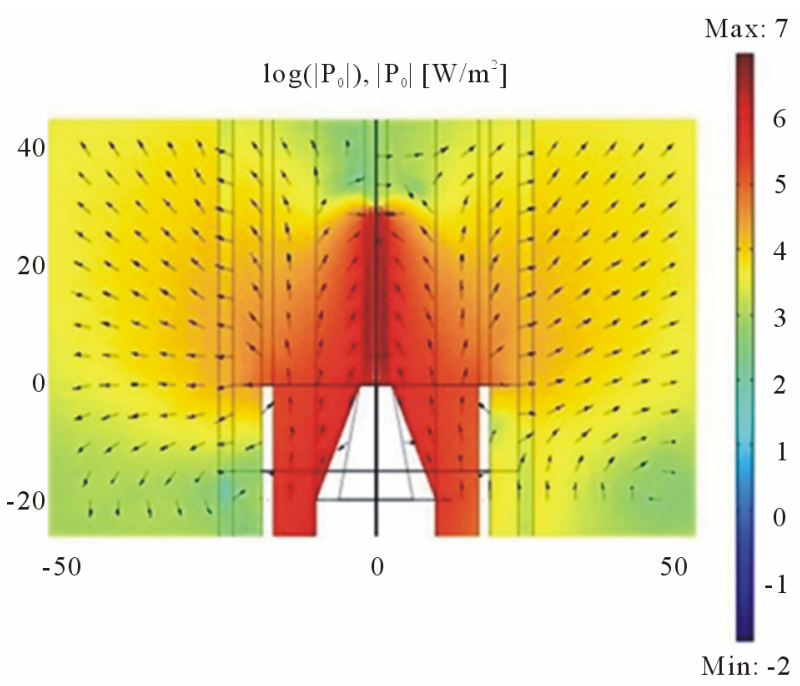

(a)

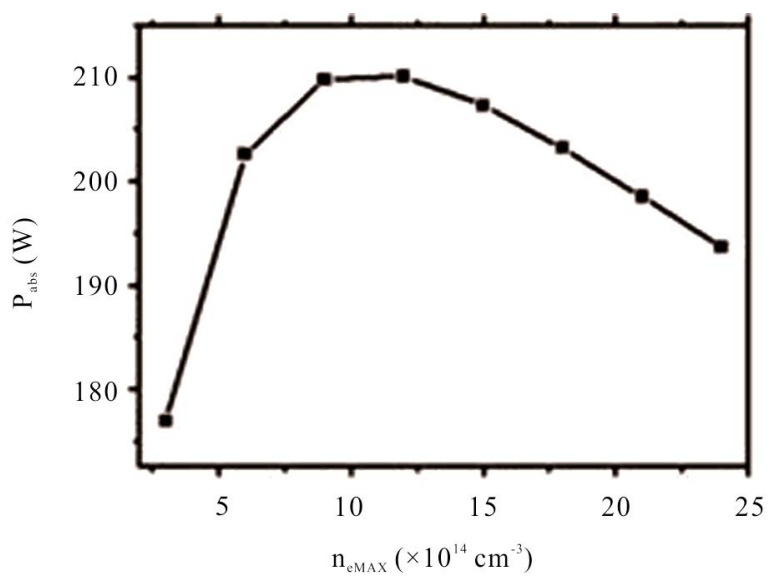

(b)

Figure 6. (a) Contour-plot $(r, z)$ in $\mathrm{mm}$, near the nozzle, of the decimal logarithm of the time-average intensity of the Poynting vector, at $n_{e \mathrm{MAX}}=3 \times 10^{14} \mathrm{~cm}^{-3}$; (b) Power absorbed by the plasma as a function of the maximum electron density. The calculations were carried out at $2.45 \mathrm{GHz}$ frequency and $500 \mathrm{~W}$ input power and for plasma dimensions of $2 \mathrm{~mm}$ diameter and $3 \mathrm{~cm}$ length. 
a function of the maximum electron density. For $n_{e \operatorname{MAX}}=$ $3 \times 10^{14}-12 \times 10^{14} \mathrm{~cm}^{-3}$ one observes an increase in $P_{a b s}$, as in this case the power absorbed by the plasma contributes to sustain the propagation medium of the wave. Above $n_{e \mathrm{MAX}}=12 \times 10^{14} \mathrm{~cm}^{-3}$ the plasma conductivity becomes comparable to that of a metal, and its behavior is similar to a radiating antenna. The competition between the power required to sustain the plasma and the power radiated by the "plasma antenna" can justify the decrease in $P_{a b s}$ as $n_{e \text { MAX }}$ varies between $12 \times 10^{14}$ and 24 $\times 10^{14} \mathrm{~cm}^{-3}[10]$. Note that the energy losses by radiation can be estimated. For instance, in the case of the $2 \mathrm{~mm}$ nozzle, for a typical electron density of $3 \times 10^{14} \mathrm{~cm}^{-3}$ we obtain at $2.45 \mathrm{GHz}$ around $40 \%$ of $500 \mathrm{~W}$ i.e. $200 \mathrm{~W}$ of coupled power (see Figure 5), and since around $175 \mathrm{~W}$ are absorbed by the plasma (see Figure 6(b)), we can deduce that $25 \mathrm{~W}$ are lost by radiation, which represents less than $15 \%$ of the coupled power.

\subsection{Hydrodynamic Module}

The HD-module was used to analyze the influence of the plasma parameters and of the inlet flow rate on the plasma-to-gas thermal transfer.

\subsubsection{Influence of Plasma Parameters}

We remind here the main effects of the plasma parameters on the HD behavior of the gas/plasma system, which have been presented previously [23]. As shown in Figure 7, the gas temperature is sensitive to changes in the electron density: $T_{g}$ increases with $n_{e}$, because the power transfer from the plasma to the gas is mainly due to electron-neutral collisions, thus becoming more effective at

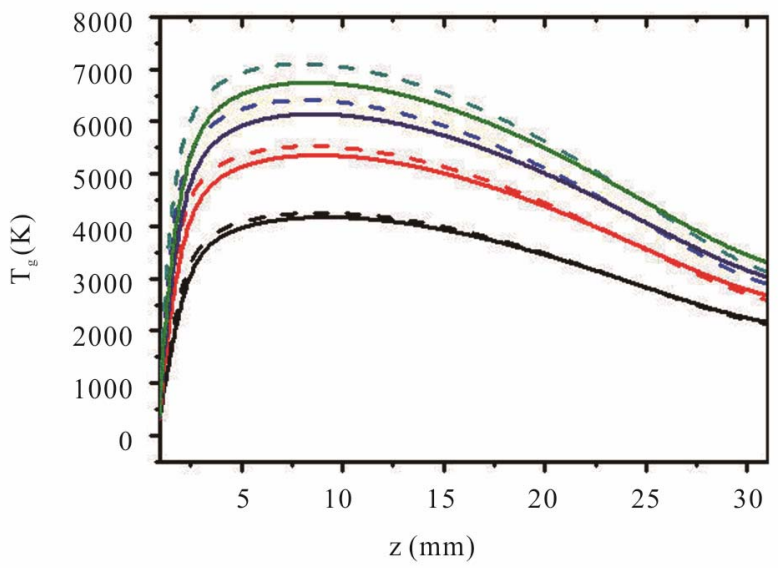

Figure 7. Axial profile (at $r=0$ ) of the gas temperature $T_{g}$ within the plasma region for the $2 \mathrm{~mm}$ nozzle $(30 \mathrm{~mm}$ plasma length), calculated at $5 \mathrm{~L} \cdot \mathrm{min}^{-1}$ input gas flow, for $T_{e \text { MAX }}=2 \times 10^{4} \mathrm{~K}$ and the following $n_{e \text { MAX values (in } 10^{14}}$ $\mathrm{cm}^{-3}$ ): 3 (black curve), 6 (red), 9 (blue) and 12 (green). The solid (dashed) curves correspond to profiles calculated with (without) the ion drift terms in the HD equations. high energy-density exchange rates (see last term of Equation (ix) in the Appendix, depending on the $n_{e} T_{e} v$ product). The results in Figure 7 show also that introducing the ion drift terms in the equations of the HDmodule (see last terms of Equations (xii) and (xiii) in the Appendix) reduces the gas temperature, due to a decrease in the residence time of the heavy species for heat exchange.

\subsubsection{Influence of Inlet Flow Rate}

Figure 8 analyses the influence on $T_{g}$ of the gas inlet flow rate, for the nozzle diameters adopted in this work. According to our experimental observations, the input

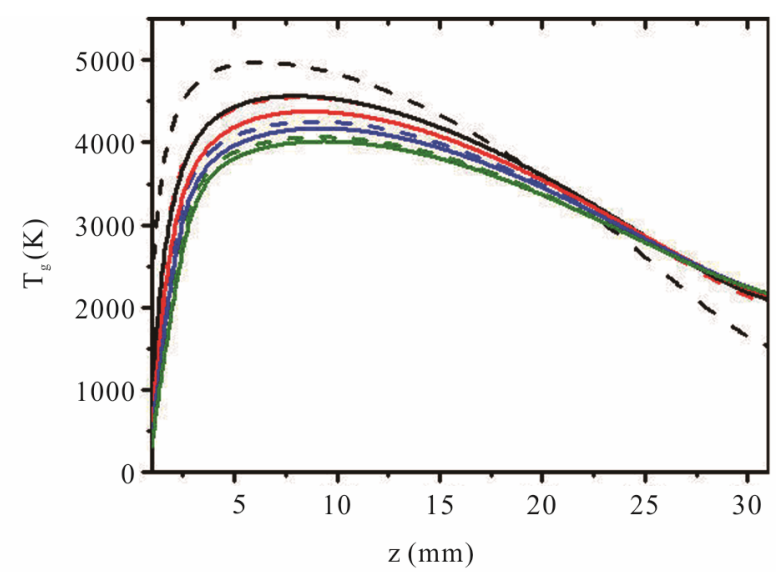

(a)

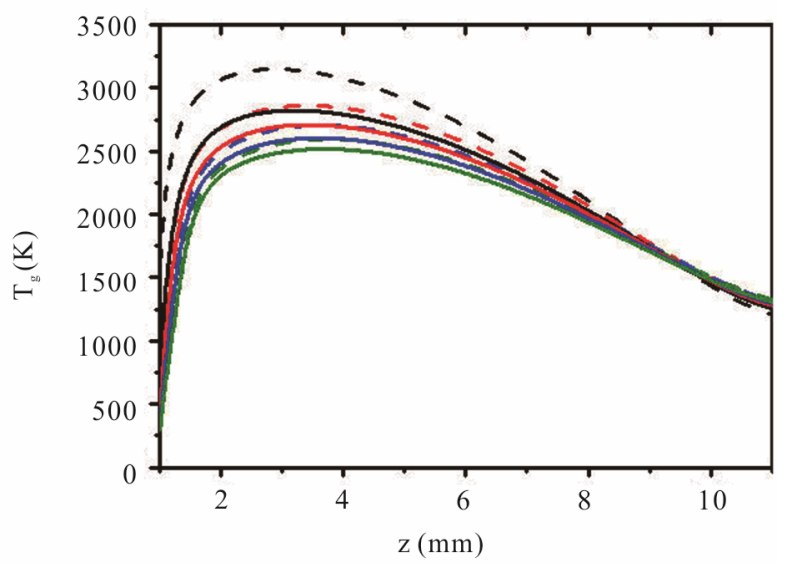

(b)

Figure 8. Axial profile (at $r=0$ ) of the gas temperature $T_{g}$ within the plasma region, (a) calculated for the $2 \mathrm{~mm}$ diameter nozzle (30 mm plasma length) at $n_{e \mathrm{MAX}}=3 \times 10^{14}$ $\mathrm{cm}^{-3}, T_{\text {MAX }}=2 \times 10^{4} \mathrm{~K}$ and the following input gas flows (in $\mathbf{L} \cdot \mathrm{min}^{-1}$ ): 1 (black curves), 3 (red), 5 (blue) and 7 (green); (b) calculated for the $0.5 \mathrm{~mm}$ diameter nozzle $(10 \mathrm{~mm}$ plasma length) at $n_{e \mathrm{MAX}}=12 \times 10^{14} \mathrm{~cm}^{-3}, T_{e \mathrm{MAX}}=2 \times 10^{4} \mathrm{~K}$ and the following input gas flows (in $\mathrm{L} \cdot \mathrm{min}^{-1}$ ): 0.25 (black curves), 0.5 (red), 0.75 (blue) and 1 (green). The solid (dashed) curves correspond to profiles calculated with (without) the ion drift terms in the HD equations. 
gas flows are lower (see Section 4.1), the maximum electron density is higher and the plasma length is shorter for the smaller diameter nozzle.

By analyzing each Figures 8(a) and (b) one concludes that an increase in the input gas flow reduces the efficiency of heat transfer between the gas and the plasma, leading to longer relaxation lengths in the axial direction for the gas temperature profile and to a reduction in its maximum value. For the fixed $n_{e}$ and $T_{e}$ profiles considered, the maximum gas temperature in the axial direction is located around $8-10 \mathrm{~mm}$ for the $2 \mathrm{~mm}$ diameter nozzle and near 3 - $4 \mathrm{~mm}$ for the $0.5 \mathrm{~mm}$ diameter nozzle, after the position of $n_{e \operatorname{MAX}}$ (at $z=1 \mathrm{~mm}$, see Appendix).

By comparing Figures 8(a) and (b) one observes that the $2 \mathrm{~mm}$ diameter nozzle allows obtaining higher gas temperatures, although the density is lower for this nozzle. This low $n_{e \text { MAX }}$ value is compensated by the lower relaxation lengths (lower gas velocity, see Table 1) in this case even if the gas flow is higher, due to larger nozzle diameter, yielding an overall increase in $T_{g}$. This point will be discussed in the experimental temperature results (Section 5.3).

These observations show that the HD features of the gas/plasma system, and particularly the definition of the gas temperature, are the combined result of local electron heating (via electron-neutral collisions) and non-local heat transport (via convection and ion drift effects). Note that ion drift effects are more important at low input gas flows, for which the relative influence of these terms is enhanced.

\subsection{Experimental Gas Temperature}

Figure 9 presents experimental values of $T_{g}$, obtained by OES of the ro-vibrational band of nitrogen near the nozzle (see Section 4.2), as a function of the input gas flow for various input powers and for the nozzle diameters considered here. An observation of this figure reveals that: 1) the gas temperature is higher when the TIA operates with the larger $2 \mathrm{~mm}$ diameter nozzle; 2) $T_{g}$ decreases when the flow increases and/or the input power decreases. The latter results are obvious for the $0.5 \mathrm{~mm}$ diameter nozzle, and can be drawn also for the $2 \mathrm{~mm}$ one at low input gas flows, in which case there is less competition between the effects of the flow and the input power (or the electron density) to define the gas temperature value.

The previous observations are in qualitative agreement with the simulation results presented in Section 5.2.2, the differences between the calculated and the measured $T_{g}$ absolute values being motivated (at least partially) by the use of imposed plasma profiles in the simulations. This quantitative disagreement can introduce also a discussion about the experimental results, beyond the mere obser- vation that measurements have a significant uncertainty, confirmed by the error bars depicted in Figure 9. In fact, measurements use the nitrogen dragged into the plasma jet from the air envelope which surrounds it, meaning that probably the experimental gas temperature does not accurately account for the plasma-to-gas heat transfer occurring near the discharge axis. Note that our intention here was to probe the plasma as it is created, thus with the molecular species coming from $\mathrm{O}_{2}, \mathrm{~N}_{2}, \ldots$, whose presence is expected to introduce a significant difference between the OES-diagnosed ro-vibrational temperature and the pure helium gas temperature [24].

Figure 10 plots the measured gas temperature as a function of the average input gas velocity, for the two nozzle diameters adopted in this work. As mentioned in Section 5.2.1, the gas temperature values are very sensitive to the residence time of the heavy species for heat exchange. Therefore, an increase in the gas velocity (due to an increase in the flow and/or a reduction in the nozzle

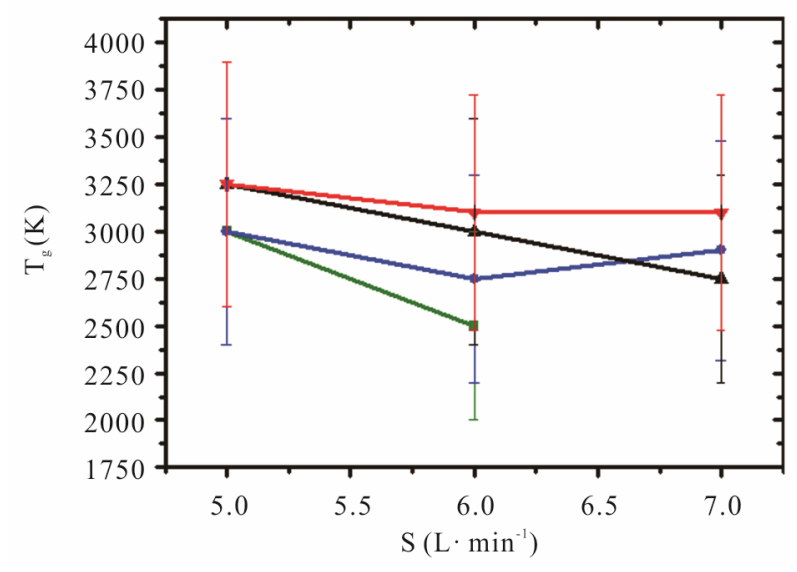

(a)

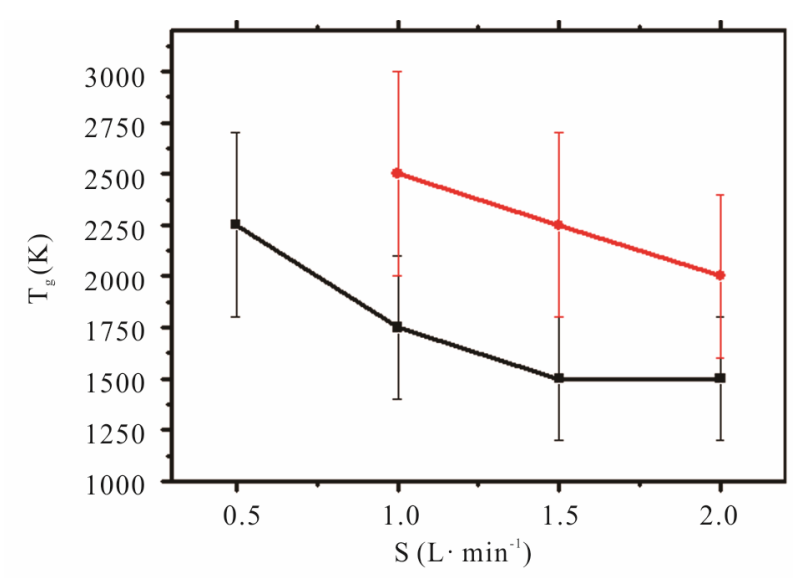

(b)

Figure 9. Measured gas temperature $T_{g}$ vs. input gas flow $S$, for TIAs with nozzle diameters of $2 \mathrm{~mm}$ (a) and $0.5 \mathrm{~mm}$ (b), obtained for the following $P_{G}$ values (in W): 400 (green curves), 500 (blue), 600 (black) and 700 (red). 


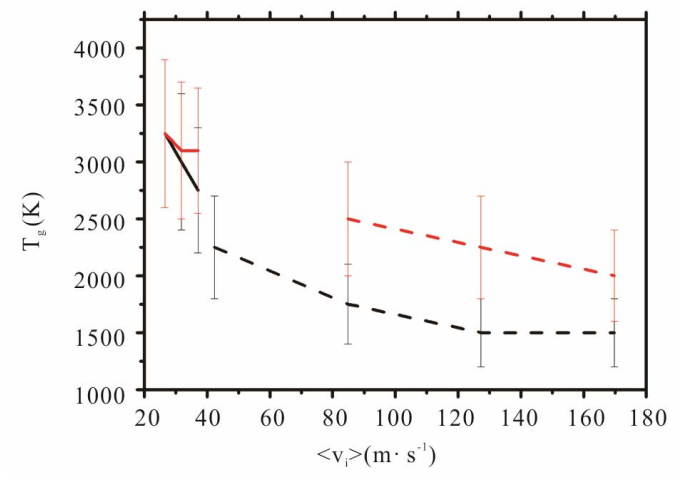

Figure 10. Measured gas temperature $T_{g}$ vs. average input gas velocity $\left\langle v_{i}\right\rangle$, for TIAs with nozzle diameters of $0.5 \mathrm{~mm}$ (dashed lines) and $2 \mathrm{~mm}$ (solid lines) for microwave powers of $600 \mathrm{~W}$ (black) and $700 \mathrm{~W}$ (red).

diameter, see Table 1), is responsible for a decrease in the residence time (typically of $\sim 1 \mathrm{~ms}$ and $\sim 0.1 \mathrm{~ms}$ for the $2 \mathrm{~mm}$ and $0.5 \mathrm{~mm}$ nozzle diameters, respectively, at 5 and $1 \mathrm{~L} \cdot \mathrm{min}^{-1}$ input gas flow), thus for a reduction in $T_{g}$ as observed in Figure 10.

\section{Conclusions}

The work presented in this paper was devoted to the study of plasmas created by microwave $(2.45 \mathrm{GHz}$ frequency) axial injection torches (TIA), running in atmospheric helium in air, in order to study the plasma-to-gas energy transfer for gas heating. The work adopted two complementary approaches based on simulations and experiments.

The TIA is a very complex discharge due to the many parameters involved in its operation: dimensions, gas flow, power input. The plasma obtained is not in thermodynamic equilibrium and is very inhomogeneous. In addition, it interacts with the surrounding external medium (air), even if the plasma is launched into some confinement chamber, such as a quartz tube. The present studies were conducted to maximize the coupling between the microwave power and the plasma and to define the optimum plasma and flow operating conditions for plasma-to-gas heat transfer.

Modeling involved the development of electromagnetic (EM) and hydrodynamic (HD) models to analyze the influence of the plasma parameters on the microwave power absorbed by the plasma and on the gas temperature.

Experiments analyzed the torch efficiency and stability, as a function of both the input power and the input gas flow, providing a framework of conditions for the simulations. Values for the plasma parameters (electron density and temperature, used as input data to the model) and for the gas temperature (also at the wall, used as boundary condition in the model) were obtained mainly from optical emission spectroscopy diagnostics.
Results yielded a good coupling efficiency $(>90 \%)$ of the TIA for input powers of 400-800 W (corresponding to electron densities of $10^{14}-10^{15} \mathrm{~cm}^{-3}$ and estimated electron temperatures of 17,000 - 25,000 K) and for input gas flows of $5-9 \mathrm{~L} \cdot \mathrm{min}^{-1}$ (for a nozzle with $2 \mathrm{~mm}$ in diameter) and of $1-3 \mathrm{~L} \cdot \mathrm{min}^{-1}$ (for another with $0.5 \mathrm{~mm}$ ). Under these conditions, simulations showed that the electron density affects both the power absorbed by the plasma and the gas temperature, the latter being enhanced by higher electron energy densities transferred to the gas by electron-neutral collisions. It was also found that by reducing the input gas velocity one increases the residence time of heavy species for collisional heat exchange, thus increasing the gas temperature. The TIA with the $2 \mathrm{~mm}$ diameter nozzle, for which the gas velocities are lower (and thus the residence time of species is longer), favors gas heating although the electron density is lower in this case.

A comparison between calculations and measurements of the gas temperature provided good qualitative agreement for the influence of the plasma parameters and the gas flow on results. However, model predictions for the gas temperature overestimate its measurements probably due to a combination of different reasons, such as: 1) the dimensions and parameters characterizing the plasma (density and temperature) were not self-consistently calculated, and remained fixed for the different work conditions considered (microwave power and gas flow); 2) the experimental gas temperatures were obtained from the ro-vibrational spectrum of the second positive system of $\mathrm{N}_{2}$, dragged into the helium plasma jet from its surrounding air envelop. The model evaluates the gas temperature at the plasma center (assumed hotter) and does not account for the incorporation of molecular species (which may decrease the gas temperature value).

Overall, the present work reveals the high potential of microwave TIAs for gas heating, demonstrating the feasibility of its stable operation, at low input powers and moderate gas flows, to obtain gas temperatures of 3000 $4000 \mathrm{~K}$.

\section{REFERENCES}

[1] C. Tendero, C. Tixier, P. Tristant, J. Demaison and P. Leprince, "Atmospheric Pressure Plasmas: A Review," Spectrochimica Acta Part B: Atomic Spectroscopy, Vol. 61, No. 1, 2006, pp. 2-30. doi:10.1016/j.sab.2005.10.003

[2] N. Azouz, S. Chaabani, J. Lerbet and A. Abichou, "Computation of the Added Masses of an Unconventional Airship," Journal of Applied Mathematics, Vol. 2012, 2012, Article ID: 714627.

[3] R. B. Bird, W. E. Steward and E. N. Lightfoot, "Transport phenomena," John Wiley, Hoboken, 1960.

[4] P. Fauchais and A. Vardelle, "Thermal Plasmas," IEEE 
Transactions on Plasma Science, Vol. 25, No. 6, 1997, pp. 1258-1280. doi:10.1109/27.650901

[5] E. A. H. Timmermans, J. Jonkers, I. A. J. Thomas, A. Rodero, M. C. Quintero, A. Sola, A. Gamero and J. A. M. van der Mullen, "The Behavior of Molecules in Microwave-Induced Plasmas Studied by Optical Emission Spectroscopy. 1. Plasmas at Atmospheric Pressure," Spectrochimica Acta Part B: Atomic Spectroscopy, Vol. 53, No. 11, 1998, pp. 1553-1566. doi:10.1016/S0584-8547(98)00186-4

[6] M. Moisan, Z. Zakrzewski, R. Pantel and P. Leprince, "A Waveguide-Based Launcher to Sustain Long Plasma Columns through the Propagation of an Electromagnetic Surface-Wave," IEEE Transactions on Plasma Science, Vol. 12, No. 3, 1984, pp. 203-214. doi:10.1109/TPS.1984.4316320

[7] M. Moisan, G. Sauve, Z. Zakrzewski and J. Hubert, "An Atmospheric Pressure Waveguidefed Microwave Plasma Torch: The Tia Design," Plasma Sources Science \& Technology, Vol. 3, No. 4, 1994, pp. 584-592. doi:10.1088/0963-0252/3/4/016

[8] A. Ricard, L. Stonge, H. Malvos, A. Gicquel, J. Hubert and M. Moisan, "Torche à Plasma Micro-Onde: Deux Configurations Complémentaires," Journal de Physique III, Vol. 5, No. 8, 1995, pp. 1269-1285. doi:10.1051/jp3:1995185

[9] J. Torres, M. J. van de Sande, J. J. A. M. van der Mullen, A. Gamero and A. Sola, "Stark Broadening for Simultaneous Diagnostics of the Electron Density and Temperature in Atmospheric Microwave Discharges," Spectrochimica Acta Part B: Atomic Spectroscopy, Vol. 61, No. 1, 2006, pp. 58-68. doi:10.1016/j.sab.2005.11.002

[10] J. Jonkers, J. M. deRegt, J. A. M. vanderMullen, H. P. C. Vos, F. P. J. deGroote and E. A. H. Timmermans, "On the Electron Temperatures and Densities in Plasmas Produced by the 'Torche a Injection Axiale'," Spectrochimica Acta Part B: Atomic Spectroscopy, Vol. 51, No. 11, 1996, pp. 1385-1392. doi:10.1016/0584-8547(96)01493-0

[11] M. Jimenez-Diaz, J. van Dijk and J. J. A. M. van der Mullen, "Effect of Remote Field Electromagnetic Boundary Conditions on Microwave-Induced Plasma Torches," Journal of Physics D: Applied Physics, Vol. 44, No. 16, 2011, Article ID: 165203 . doi:10.1088/0022-3727/44/16/165203

[12] L. L. Alves, R. Alvarez, L. Marques, S. J. Rubio, A. Rodero and M. C. Quintero, "Modeling of an Axial Injection Torch," European Physical Journal: Applied Physics, Vol. 46, No. 2, 2009, p. 21001. doi:10.1051/epjap/2009049

[13] E. A. H. Timmermans, F. P. J. de Groote, J. Jonkers, A. Gamero, A. Sola and J. J. A. M. van der Mullen, "Atomic Emission Spectroscopy for the On-Line Monitoring of Incineration Processes," Spectrochimica Acta Part B: Atomic Spectroscopy, Vol. 58, No. 5, 2003, pp. 823-836. doi:10.1016/S0584-8547(03)00017-X

[14] S. S. Asad, C. Tendero, C. Dublanche-Tixier, P. Tristant, C. Boisse-Laporte, O. Leroy and P. Leprince, "Effect of Atmospheric Microwave Plasma Treatment on Organic Lubricant on a Metallic Surface," Surface \& Coatings Technology, Vol. 203, No. 13, 2009, pp. 1790-1796. doi:10.1016/j.surfcoat.2008.12.026
[15] S. J. Rubio, A. Rodero and M. C. Quintero, "Application of a Microwave Helium Plasma Torch Operating at Atmospheric Pressure to Destroy Trichloroethylene," Plasma Chemistry and Plasma Processing, Vol. 28, No. 4, 2008, pp. 415-428. doi:10.1007/s11090-008-9133-3

[16] L. L. Alves and C. M. Ferreira, "Electron Kinetics in Weakly Ionized Helium under DC and HF Applied Electric-fields," Journal of Physics D: Applied Physics, Vol. 24, No. 4, 1991, pp. 581-592. doi:10.1088/0022-3727/24/4/009

[17] http://www.comsol.com

[18] C. O. Laux, T. G. Spence, C. H. Kruger and R. N. Zare, "Optical Diagnostics of Atmospheric Pressure Air Plasmas," Plasma Sources Science \& Technology, Vol. 12, 2003, pp. 125-138.

[19] M. A. Gigosos and V. Cardenoso, "New Plasma Diagnosis Tables of Hydrogen Stark Broadening Including Ion Dynamics," Journal of Physics B: Atomic Molecular and Optical Physics, Vol. 29, No. 20, 1996, pp. 4795-4838. doi:10.1088/0953-4075/29/20/029

[20] M. A. Gigosos, M. A. Gonzalez and V. Cardenoso, "Computer Simulated Balmer-Alpha, -Beta and -Gamma Stark Line Profiles for Non-Equilibrium Plasmas Diagnostics," Spectrochimica Acta Part B: Atomic Spectroscopy, Vol. 58, No. 8, 2003, pp. 1489-1504. doi:10.1016/S0584-8547(03)00097-1

[21] J. Torres, O. Carabano, M. Fernandez, S. Rubio, R. Alvarez, A. Rodero, C. Lao, M. C. Quintero, A. Gamero and A. Sola, "The Stark-Crossing Method for the Simultaneous Determination of the Electron Temperature and Density in Plasmas," Journal of Physics Conference Series, Vol. 44, No. 1, 2006, p. 70. doi:10.1088/1742-6596/44/1/008

[22] M. A. Heald and C. B. Wharton, "Plasma Dignotics with Microwave," Wiley, New York, 1965.

[23] K. Gadonna, O. Leroy, T. Silva, P. Leprince, C. BoisseLaporte and L. L. Alves, "Hydrodynamic Study of a Microwave Plasma Torch," European Physics Journal of Applied Physics, Vol. 56, No. 2, 2011, p. 24008. doi:10.1051/epjap/2011110161

[24] R. P. Cardoso, T. Belmonte, P. Keravec, F. Kosior and G. Henrion, "Influence of Impurities on the Temperature of an Atmospheric Helium Plasma in Microwave Resonant Cavity," Journal of Physics D: Applied Physics, Vol. 40, No. 5, 2007, pp. 1394-1400. doi:10.1088/0022-3727/40/5/012

[25] O. Schenk and K. Gärtner, "Solving Unsymmetric Sparse Systems of Linear Equations with PARDISO," Journal of Future Generation Computer Systems, Vol. 20, No. 3, 2004, pp. 475-487.

[26] J. P. Berenger, "Three-Dimensional Perfectly Matched Layer for the Absorption of Electromagnetic Waves," Journal of Computational Physics, Vol. 127, No. 2, 1996 , pp. 363-379. doi:10.1006/jcph.1996.0181

[27] T. Belmonte, R. P. Cardoso, G. Henrion and F. Kosior, "Collisional-Radiative Modelling of a Helium Microwave Plasma in a Resonant Cavity," Journal of Physics D: Applied Physics, Vol. 40, No. 23, 2007, pp. 7343-7356. doi:10.1088/0022-3727/40/23/015 


\section{Appendix}

We present here an overview of the plasma description, the domain of calculation used in the models, and equations and boundary conditions used in 3D electromagnetic and 2D hydrodynamic models developed under the COMSOL Multiphysics platform [17].

\section{A.1. Plasma Description}

For both electromagnetic and hydrodynamic models, the plasma is defined by imposing its dimensions (length and radius) and the axial and radial profiles of $n_{e}(r, z)=$ $n_{e \mathrm{MAX}} n_{e r}(r) n_{e z}(z)$ and $T_{e}(r, z)=T_{e \mathrm{MAX}} T_{e r}(r) T_{e z}(z)$, shown in Figure 11. The axial profile of $n_{e}$ has been chosen in accordance with measurements of optical emission spectroscopy (Section 4.2). As it has been experimentally observed, the beginning of the plasma is located $1 \mathrm{~mm}$ above the nozzle. $T_{e}$ profiles are defined according to

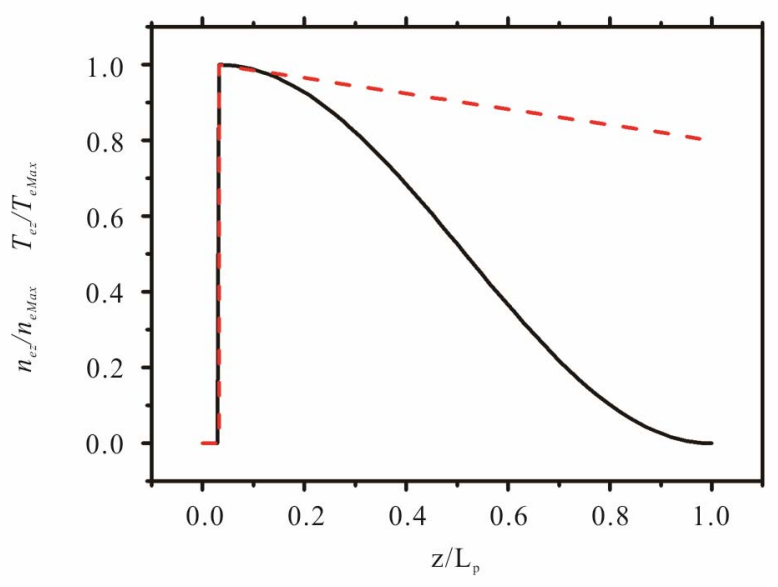

(a)

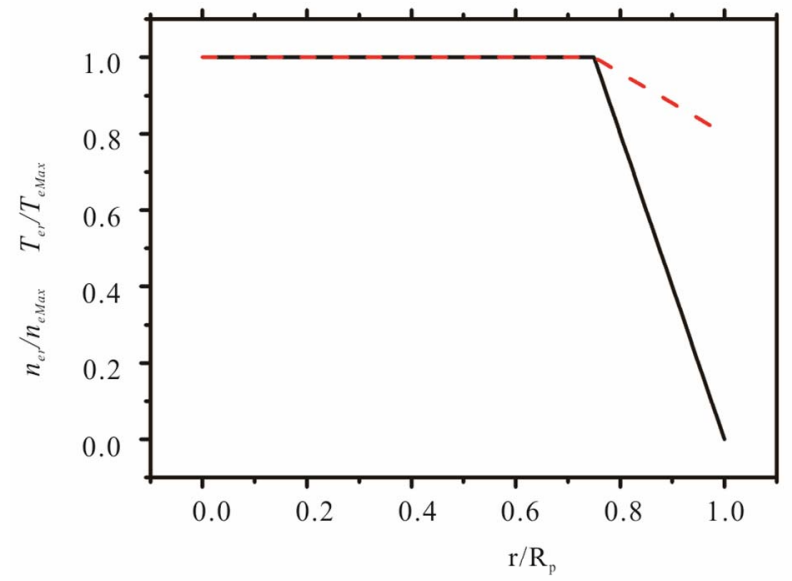

(b)

Figure 11. Axial (a) and radial (b) profiles of the electron density $n_{e}$ (black curves) and the electron temperature $T_{e}$ (red curves). $R_{p}$ and $L_{p}$ are respectively the radius and the length of the plasma.
$[9,21]$. The studied gas is helium at atmospheric pressure.

Note that $R_{p}, L_{p}, n_{e \mathrm{MAX}}$ and $T_{e \mathrm{MAX}}$ are input parameters of the model. For all the simulations, we chose to fix $R_{p}$ equal to the nozzle radius and to take $L_{p}$ in accordance with the experiment. Typically $L_{p}=30 \mathrm{~mm}$ for the $2 \mathrm{~mm}$ nozzle and $L_{p}=10 \mathrm{~mm}$ for the $0.5 \mathrm{~mm}$ nozzle.

\section{A.2. Domain of Calculation}

Figure 12 shows the computational domain on which the EM and HD modules are solved. All the dimensions introduced in the model are the same than the ones used in our experiments.

For the EM module, we chose to build a 3D model of the system to evaluate the coupling between the rectangular waveguide, the coaxial transition and the plasma. The tetrahedral meshing uses around $8 \times 10^{4}$ elements for the whole computational domain with a refinement at the nozzle exit, and in the plasma (typically $3 \times 10^{3}$ elements).

For the HD module, the plasma gas is injected with a given velocity. We assumed (see Table 1) that the flow remains laminar, which reduces the computation time by adopting the axisymmetric $2 \mathrm{D}$ geometry in HD module. The triangular meshing uses around $4 \times 10^{4}$ elements and is refined in the plasma.

The solver used is PARDISO [25]. This direct solver is widely used because of its ease in use and its robustness: it does not need preconditioner. The convergence criterion imposes relative errors between consecutive calculations, typically less than $10^{-6}$.

\section{A.3. Electromagnetic Module}

The distribution of electromagnetic field in the presence of the plasma is calculated by solving the MaxwellAmpere and Faraday-Maxwell equations:

$$
\begin{gathered}
\nabla \times \boldsymbol{H}=\sigma \boldsymbol{E}+\frac{\partial(\varepsilon \boldsymbol{E})}{\partial t} \\
\nabla \times \boldsymbol{E}=-\mu \frac{\partial \boldsymbol{H}}{\partial t}
\end{gathered}
$$

$\boldsymbol{E}$ is the electric field $[\mathrm{V} / \mathrm{m}], \boldsymbol{H}$ is the magnetic field $[\mathrm{A} / \mathrm{m}], \varepsilon$ is the permittivity $[\mathrm{F} / \mathrm{m}], \mu$ is the permeability $[\mathrm{H} / \mathrm{m}]$ and $\sigma$ is the conductivity of the medium $[\mathrm{S} / \mathrm{m}]$.

By writing $\boldsymbol{E}(x, y, z, t)=\boldsymbol{E}(x, y, z) \exp (j \omega t)$ and $\boldsymbol{H}(x, y$, $z, t)=\boldsymbol{H}(x, y, z) \exp (j \omega t)$, the two laws can be combined to obtain the electric field wave equation:

$$
\nabla \times\left(\frac{1}{\mu_{r}} \nabla \times \boldsymbol{E}\right)-k_{0}^{2}\left(\varepsilon_{r}-\frac{j \sigma}{\omega \varepsilon_{0}}\right) \boldsymbol{E}=0
$$

with $\mu=\mu_{0} \mu_{r}$ and $\varepsilon=\varepsilon_{0} \varepsilon_{r}$, where $\mu_{0}$ is the vacuum permeability and $\varepsilon_{0}$ the vacuum permittivity. The relative 


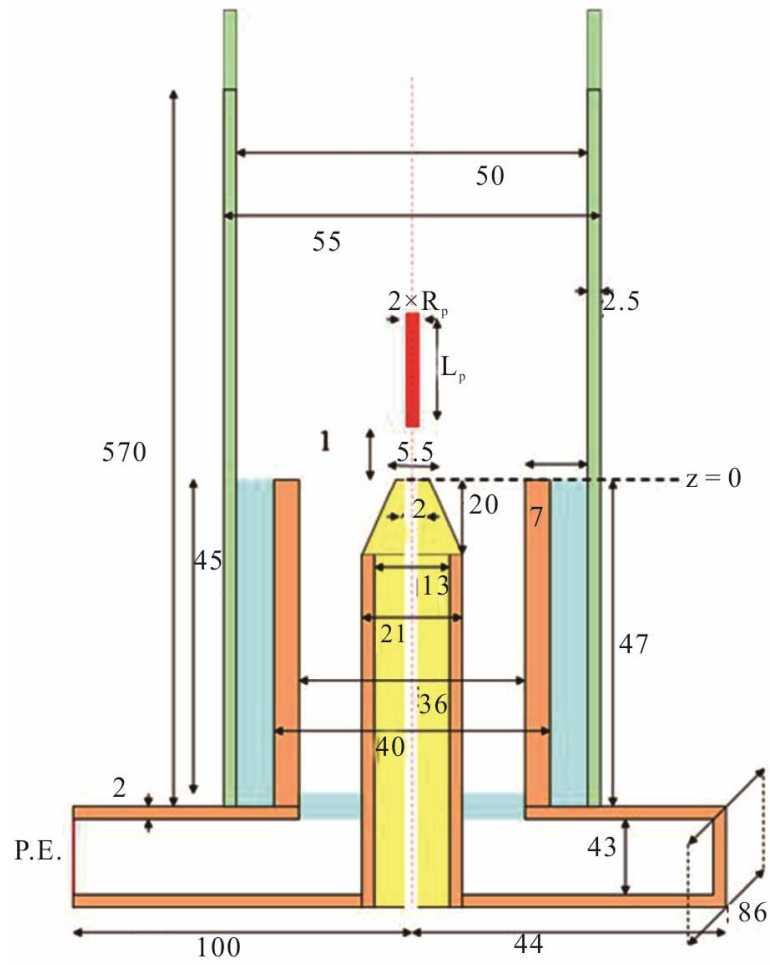

(a)

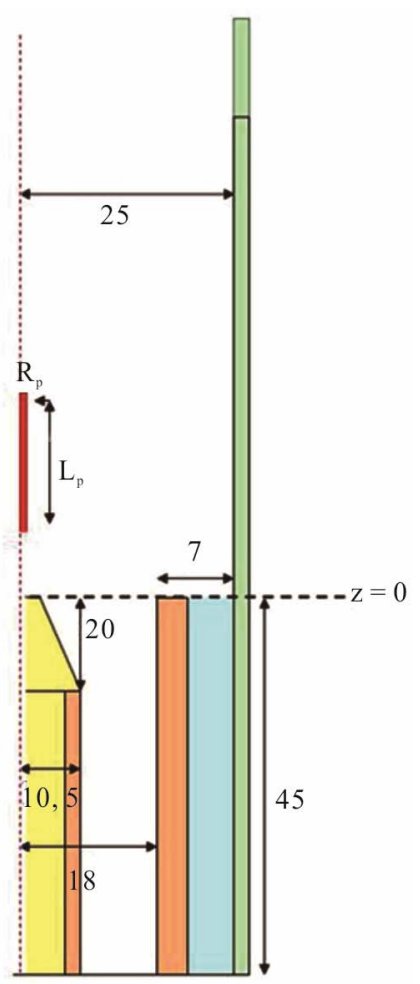

(b)

Figure 12. Transverse section of the 3D (a) and 2D (b) computational domains adopted to solve the EM and HD modules, showing the waveguides (in brown), the torch (in yellow), the quartz tube (in green), Teflon (in blue) and the plasma (in red). All dimensions are in millimeters and the diagram is not to scale. The plasma is located $1 \mathrm{~mm}$ above the nozzle.

permittivity $\varepsilon_{r}$, the relative permeability $\mu_{r}$ and the conductivity $\sigma$ define the different environments. $k_{0}$ is the wave number in vacuum.

In these equations, the plasma is considered as a vacuum environment, having a conductivity due to free charges (electrons), i.e.:

$$
\begin{gathered}
\varepsilon_{r p}=1 \\
\sigma=\sigma_{p}=\frac{n_{e} e^{2}}{m_{e}(v+j \omega)}
\end{gathered}
$$

The values of permittivity, permeability and conductivity of the different media used in the model are given in Table 2.

The boundary conditions for the waveguide and the torch (made of brass) are those of a perfect electrical conductor $(\boldsymbol{n} \times \boldsymbol{E}=0)$, except for the section of the guide (P.E. on Figure 12) where the microwave excitation is applied. At this point, a condition of port for a $\mathrm{TE}_{10}$ rectangular mode is imposed. Moreover, this configuration, shown in Figure 12, is surrounded by a region of PML type (Perfectly Matched Layers) [26], where boundary conditions of diffusion (non-reflective) are assumed. This consists in adding an artificial field which
Table 2. Permittivity, permeability and conductivity of air, brass, teflon, quartz and plasma.

\begin{tabular}{cccc}
\hline & $\varepsilon_{r}$ & $\mu_{r}$ & $\sigma$ \\
\hline Air & 1 & 1 & 0 \\
Brass & 1 & 1 & $1.57 \times 10^{7}$ \\
Teflon & 2.8 & 1 & $10^{-22}$ \\
Quartz & 3.8 & 1 & $10^{-18}$ \\
Plasma & 1 & 1 & $\sigma_{p}$ \\
\hline
\end{tabular}

absorbes the waves.

\section{A.4. Hydrodynamic Module}

This section focuses on the transport of heavy species, neutral or ionized, whose masses are comparable. The distributions of velocities and temperatures in the presence of the plasma are calculated by solving the wellknown Navier-Stokes equations:

$$
\begin{gathered}
\nabla \cdot\left(\rho v_{0}\right)=0 \\
\rho\left(v_{0} \cdot \nabla\right) v_{0}=-\nabla p-\nabla \cdot \tau
\end{gathered}
$$


where $\rho$ is the gas density, $\mathrm{v}_{0}$ is the gas velocity, $p$ is the gas pressure, $\boldsymbol{\tau}$ is the viscosity tensor given by:

$$
\tau=-\eta\left[\nabla v_{0}+\left(\nabla v_{0}\right)-\frac{2}{3} \nabla \cdot v_{0} I\right]
$$

where $\boldsymbol{I}$ is the identity tensor.

The viscosity tensor accounts for the dynamic viscosity coefficient of the fluid $\eta$, which can be obtained by the Chapman-Enskog theory using Lennard-Jones parameters [3].

The combination of the continuity Equation (vi) and the momentum Equation (vii) provides a description of the barycentric velocity, in terms of pressure gradient and viscosity.

The gas flow is then completed by heat transfer using the equation of energy conservation given by:

$$
\begin{aligned}
& \rho C_{v}\left(\boldsymbol{v}_{0} \cdot \nabla\right) T_{g} \\
& =\nabla \cdot\left(\lambda_{g} \nabla T_{g}\right)-p \nabla \boldsymbol{v}_{0}+3 \frac{m_{e}}{M_{g}} n_{e} v k_{B}\left(T_{e}-T_{g}\right)
\end{aligned}
$$

where $k_{B}$ is the Boltzmann constant, $C_{v}$ is the specific heat of gas at constant volume, $\lambda_{g}$ is the thermal conductivity and $v$ is the electron-neutral collision frequency. $m_{e}$, $n_{e}$ and $T_{e}$ are respectively the electron mass, density and temperature. $\lambda_{g}$ depends on $\eta$ [3] as following:

$$
\lambda_{g}=\frac{15}{4} \eta \frac{k_{B}}{M_{g}}
$$

where $M_{g}$ is the mass of the gas neutrals (here helium).

Each term in Equation (ix) describes the different means of energy exchange, i.e. from left to right of equation:

- convective processes (density difference, temperature difference);

- heat conduction processes (collisions between particles of the same type);

- work of pressure forces;

- collisions between different particles (here heating of helium gas by the electrons).

The energy Equation (ix) describes and predicts the temperature of the flowing gas. It is coupled to the Navier-Stokes Equations (vi) and(vii), which describe the velocity fields. This coupling occurs through the convective term and the work of pressure forces that involve velocities. The Navier-Stokes equations are also coupled to the energy equation because the gas properties (density, viscosity) depend on temperature.

All these equations have been established without taking into account the helium ions whose mass is of the same order than neutrals. As the ionisation degree is not negligible in MPTs, we add the influence of ions on the transport of heavy particles and the heat transfer to gas.
Equations (vi), (vii) and (ix) are then rewritten as following:

$$
\begin{gathered}
\nabla \cdot\left(\rho \boldsymbol{v}_{0}\right)=0 \\
\rho\left(\boldsymbol{v}_{0} \cdot \nabla\right) \boldsymbol{v}_{0}=-\nabla p-\nabla \cdot \boldsymbol{\tau}+e n_{i} \boldsymbol{E}_{d c} \\
\rho C_{v}\left(\boldsymbol{v}_{0} \cdot \nabla\right) T_{g}=\nabla \cdot\left(\lambda_{g} \nabla T_{g}\right)-p \nabla \boldsymbol{v}_{0} \\
+3 \frac{m_{e}}{M_{g}} n_{e} \nu k_{B}\left(T_{e}-T_{g}\right)+e \boldsymbol{\Gamma}_{\mathrm{i}} \cdot \boldsymbol{E}_{d c}
\end{gathered}
$$

The ions influence occurs in the momentum equation (Equation (xii)) with the ionic force term $e n_{i} \boldsymbol{E}_{d c}$ and in the energy equation (Equation (xiii)) with the Joule heating term $e \boldsymbol{\Gamma}_{i} \cdot \boldsymbol{E}_{d c}$.

$\boldsymbol{E}_{d c}, n_{i}$ and $\boldsymbol{\Gamma}_{i}$ are respectively the space charge field, the ion density and the ion flow. They are calculated for ambipolar conditions:

$$
\begin{gathered}
\boldsymbol{E}_{d c} \approx-\frac{k_{B} T_{e}}{e} \frac{\nabla n_{e}}{n_{e}} \\
n_{i} \approx n_{e} \\
\boldsymbol{\Gamma}_{\mathrm{i}} \approx-D_{a} \nabla_{n_{e}}
\end{gathered}
$$

with

$$
D_{a} \approx\left(k_{B} T_{e} / e\right) u_{i}
$$

the ambipolar diffusion coefficient and $\mu_{i} N$ the reduced ion mobility.

In high temperature gas, both $\mathrm{He}^{+}$and $\mathrm{He}_{2}^{+}$are present $\left(\left[\mathrm{He}_{2}^{+} / \mathrm{He}^{+}\right] \sim 0.55\right.$ [27]). For simplification and as a first approach we consider a single type of ion, $\mathrm{He}_{2}{ }^{+}$ $\left(\mu_{i} \mathrm{~N}=4.49 \times 10^{20} \mathrm{~V}^{-1} \cdot \mathrm{cm}^{-1} \cdot \mathrm{s}^{-1}[16]\right)$.

Equations (xi)-(xiii) are solved in order to study the heat transfer from the plasma to the gas taking into ac-

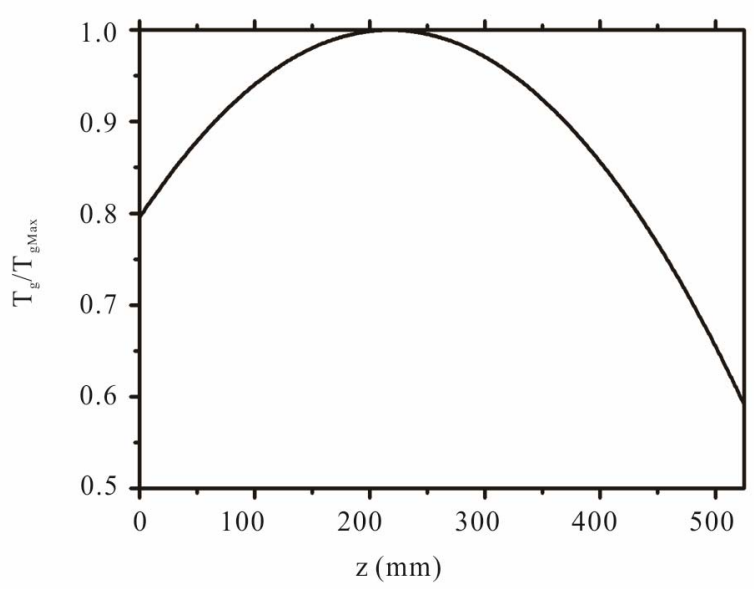

Figure 13. Temperature profile imposed at $r=25 \mathrm{~mm}$. For $0 \mathrm{~mm} \leq z \leq 525 \mathrm{~mm}: T_{g}(z)=0.8+1.9 \mathrm{z}[\mathrm{m}]-4.3 \mathrm{z}[\mathrm{m}]^{2}$. For $z$ $\geq 525 \mathrm{~mm}, T_{g}=290 \mathrm{~K}$. 
count the influence of ions. We compared these results with the ones obtained without the ions terms.

The boundary conditions used to solve the HD-module are the following:

- on the axis $(r=0)$ : axisymmetric conditions;

- $\boldsymbol{n} \cdot(-k \nabla T)$ (convective flux) on the metallic parts of the torch;

- $v=0$ ("no-slip" condition) on all the walls;

- At the entrance $(z=-45 \mathrm{~mm}$, bottom left of Figure 12.b)), $T_{g}$ is considered equals to room temperature $(290 \mathrm{~K})$ and the gas velocity is given by:

$$
v_{z=-45}(r)=2\left\langle v_{i z}\right\rangle\left[1-\left(\frac{r}{R_{p}}\right)^{2}\right]=0
$$

- At the exit (at the exit of the quartz tube $z=(570-45)$ $=525 \mathrm{~mm}$ ): $p=1 \mathrm{~atm}$ and $T_{g}=290 \mathrm{~K}$;

- Temperature of the quartz tube, taken as boundary value for $T_{g}$ at $r=25 \mathrm{~mm}$, has been experimentally measured using a thermocouple (Section 4.2) all along the tube length $(570-45)=525 \mathrm{~mm}$. It has been found that this profile is quite the same for all experimental conditions. A unique profile has been then used as boundary condition for $T_{g}$ at $r=25 \mathrm{~mm}$. This profile is shown in Figure 13. The $T_{g \text { Max }}$ value is varying with experimental conditions. But, as it has been found that it has not a great influence on model results, it has been fixed to $600 \mathrm{~K}$. 